\title{
Low back pain among nurses working in a clinical settings of Africa: A systematic review and meta-analysis of a 19 years of studies.
}

\section{Ayele Semachew ${ }^{\ddagger 1}$, Yinager Workineh², Emiru Ayalew ${ }^{2 *}$, Worku Animaw ${ }^{3}$,}

${ }^{1}$ Department of Adult Health Nursing, College of Medicine and Health science, Bahir Dar University, Ethiopia

${ }^{2}$ Department of child health Nursing, College of Medicine and Health science, Bahir Dar University, Ethiopia

$2^{*}$ Department of Adult Health Nursing, College of Medicine and Health science, Bahir Dar University, Ethiopia

${ }^{3}$ Department of Adult Health Nursing, College of Medicine and Health science, Bahir Dar University, Ethiopia

${ }^{\ddagger}$ Corresponding author

Email addresses:

Ayele Semachew (AS): ayele.semachew@yahoo.com or finoteayu24@gmail.com

Yinager Workineh (YW): workieyene@gmail.com

Emiru Ayalew (EA): emruayalew2010@gmail.com

Worku Animaw (WA): workmaw@gmail.com 


\begin{abstract}
Introduction: Because of the nature of the work, healthcare providers are prone to develop different musculoskeletal problems including low back pain and hospital healthcare workers are groups of healthcare workers who suffered a lot from it. The incidence varies between countries and professions. The situation is somewhat worsen among the frontline healthcare provider in many healthcare facilities. Nurses in Africa are arguably the most important frontline health care workers available in most African healthcare facilities, performing a broad range of tasks and working in settings where no other health workers, including physicians, are available. This situation is considerably important in the causation of work load. Nursing is listed among the highly risky profession for the development of low back pain and has been ranked with in the top tenth professions which have a great risk of having susceptible to low back pain.
\end{abstract}

Objective: The aim of this systematic review and meta-analysis was to ascertain whether LBP is of a significant concern among nurses in African healthcare facilities.

Methods: A comprehensive literature search of different data bases with no date limit was conducted from September to November 2018 using the PRISMA guideline. The quality of the included studies were assessed using a 12-item rating system. Subgroup and sensitivity analysis were performed. Cochran's Q and the $\mathrm{I}^{2}$ test were used to assess heterogeneity. The presence of publication bias was evaluated by using Egger's test and visual inspection of the symmetry in funnel plots.

Result: During the period 2000-2018, nineteen studies with a sample size of 6110 have been carried out. Among them, the lowest and the highest prevalence were found to be $44.1 \%$ and $82.7 \%$. Both the highest and the lowest prevalence of low back pain were reported from a studies done in Nigeria. The estimation of the prevalence rate of low back pain among nurses using the 
random effects model was found to be 64.07\% (95\% CI: 58.68-69.46; P-value < 0.0001). Heterogeneity of the reviewed studies was $\mathrm{I}^{2}=94.2 \%$ and heterogeneity Chi-squared $=310.06$ (d.f $=18)$, P-value $<0.0001$. The subgroup analyses showed that the highest prevalence of LBP among nurses was from west African region with prevalence rates of 68.46\% (95\% CI: 54.94- 81.97; Pvalue $<0.0001)$ and followed by north Africa region with prevalence rate of $67.95 \%$ (95\% CI: 55.96-79.94; P value <0.0001) had the higher prevalence of LBP as compared to their south African counterparts, 59\% (95\% CI: 51-66.9; P-value <0.0001).

Conclusion: Even though the overall prevalence of the present study is lower when compared to the western and Asian studies, it indicated that the prevalence of low back pain among nurses is on the move.

Key words: Low back pain, nurses, Africa, Musculoskeletal problems, back hygiene. 


\section{Introduction}

Low back pain (LBP) is one of the most common causes of musculoskeletal disorders related to work status (1). LBP is a rampant health problem responsible for serious suffering and disability than any other health complaint across the globe (2). It is estimated that LBP may be experienced as much as $80 \%$ in different population groups at some time in their lives (3-7). LBP has been shown to account for an average number of disability-adjusted life years (DALYs) higher than different infectious diseases, non-communicable disease and road traffic injuries. According to the Global Burden of Disease (GBD) 2010 report, LBP was recorded among the top ten high burden diseases and injuries (8).

Because of the nature of the work, healthcare providers are prone to develop different musculoskeletal problems including LBP (9)(10)(11) and hospital healthcare workers are groups of healthcare workers who suffered a lot from it. The incidence varies between countries and professions $(12,13)$. The situation is somewhat worsen among the frontline healthcare provider in many healthcare facilities (14).

Nurses in Africa are arguably the most important frontline health care workers available in most African healthcare facilities, performing a broad range of tasks and working in settings where no other health workers, including physicians, are available (15). This situation is considerably important in the causation of work load. Nursing is listed among the highly risky profession for the development of LBP and has been ranked with in the top tenth professions which have a great risk of having susceptible to LBP (16-19). A study done in American indicated that nurses are ranked the sixth highest with regard to lose their working days from job due to LBP (20). 
In providing a care, nurses are subjected to lift and transport patients or equipment, often in difficult environment particularly in developing nations where lifting aids are not available or practicable (21-25). This process is really hard on a nurse's back(26). Biomechanical investigations reported that such movements result into high spinal stresses (27).

Lower back pain directly affects nurses' productivity at work and consequently reduces the overall amount and quality of healthcare the clients receive (28-33). In addition, LBP will have many negative impact on different aspects of the healthcare system including absence from work place, loss of optimal performance, low job satisfaction, rising medical costs and occupational disability (34). The health of nurses influences not only their job satisfaction, quality of life and desire to change careers but also quality of care and patient safety $(9,35-37)$.

LBP has been identified as one of the main causes of loss of hours and days among the working class citizens (38). Describing the extent of musculoskeletal injury in nurses, survey showed that nurses lost 750,000 days a year as a result of back pain (24).

Different epidemiological studies have been done to identify and relate possible risk factors to the occurrence of LBP among nursing staffs. They found that individual factors such as age, gender, educational level, body mass index, and psychosocial factors referring to job satisfaction, work stress, and anger have been examined and related to the occurrence of LBP. But, LBP is a complex condition with several factors contributing to its occurrence (17,39-44). Psychosocial factors (low work support from superiors and poor nurse physician communication) are stated as an important underlying factors for the development of LBP $(21,45-47)$. 
To the researchers' knowledge, there is no prior systematic review and meta-analysis reporting on the prevalence of LBP among nurses. Hence, the objective of the current review was to thoroughly evaluate peer-reviewed published studies on the reported prevalence of LBP among nurses working at different African healthcare facilities. This would help us to ascertain whether LBP is of a significant concern among nurses in African healthcare facilities. In addition, the review tried to assess the methodological relevance of the retrieved studies in the subject area and this would help in identifying chances for service improvement in the African healthcare settings.

\section{Method}

This systematic review and meta-analysis was conducted using studies that addressed low back pain among nurses working at different African healthcare facilities and the review was presented using the PRISMA guideline (48).

\section{Search Strategy}

A comprehensive search was conducted from September to November 2018 with no date limit to each data bases. Electronic searches using main sets of terms and using their combinations was performed. The first sets were the key words that describes the population under study, the next sets of terms includes the outcome of interest of the study, the other sets of terms were the settings of the study and the final sets of terms were the study area. Based on this principle, electronic data base searching was done. The search was done with the phrase/Boolean search mood from the title, abstract and keywords. Searching terms and their combinations were; ("Nursing" OR "Professional Nurses" or "Nurses") AND ("Low back pain" OR "Prevalence of low back pain" OR "Incidence of low back pain" OR "Musculoskeletal problem") AND "Hospitals" OR "Healthcare facilities” AND “Africa” OR “African countries” 
The following data bases: PubMed, ScienceDirect, Google scholar, MEDLINE, CINHAL and ProQuest were searched to search the eligible studies. In addition to the electronic database searches, a secondary search technique known as "footnote chasing" was utilized to identify additional articles for inclusion in the review.

\section{Eligibility criteria}

Primary research works that reported the prevalence of LBP among nurses working at different African healthcare facilities were included. Studies were not restricted by time of study or year of publication but they should be written or published using English language. Thesis report, dissertation, and any report proceedings/conference in the subject matter which was published in journals were included in our searches.

\section{Definitions of terms}

Musculoskeletal disorders: Any pain or discomfort in one or more limbs.

Low Back Pain (LBP): Any pain in the lower back between L1 - L5 (lumbar spine) and L5-S1 (lumbosacral joint) (49).

Prevalence of low back pain: A 12-month recall period was used for experiencing of low back pain, as this has been shown to be an appropriate time-scale in other studies (50).

Nurses: Nurses working at different hospitals of African countries.

\section{Data extraction}

To extract the data, a form was prepared that included the following variables: Author names, year of publication, country, region in the continent, setting, study design, sample size, gender, mean age, measurement, prevalence of LBP, and studies' quality score. The extraction was done by three independent researchers (AS, YW and EA). When there was disagreement 
between them, a thorough discussion was made between them and if there was still any disagreement, the fourth author (WA) was consulted.

\section{Study quality assessment}

To assess the quality of the included studies, a modified critical appraisal tool was utilized. This tool includes three methodological tests containing 12 discrete criteria for prevalence studies. From these, three questions assesses sample representativeness of the target population, six questions assesses the data quality, and the remaining three questions assesses the definition of the outcome variable. Based on this parameter, studies with at least $75 \%$ of the total score were acceptable $(49,51-53)$ to be included to the systematic review and meta-analysis (Appendix).

\section{Statistical analysis procedure}

Data analysis was performed using STATA version 11 software and P-value $\leq 0.05$ significance level was considered. The weight given to each study was assigned according to the inverse of the variance. Cochrane $\mathrm{Q}$ and $\mathrm{I}^{2}$ statistics were used to assess heterogeneity among studies. Heterogeneity was measured by $\mathrm{I}^{2}$ and divided into four categories; no heterogeneity (0\%), low $(25-50 \%)$, moderate $(50-75 \%)$, and high $(>75 \%)(54)$.

Subgroup analysis and meta-regression (the relationship between the years of the study and region in the continent with the prevalence rate) were employed to explore the cause of heterogeneity between studies. Funnel plot (Begg's test) and Egger's statistics with pseudo 95\% confidence interval was used to examine publication bias. 


\section{Result}

Until December 10, 2018 four hundred eighteen articles were identified, all records were reviewed and 361 irrelevant and duplicate studies were excluded. The full texts of the remaining 57 articles were reviewed in detail and finally 19 articles met the inclusion criteria and included to the final section of the analysis (Figure 1).

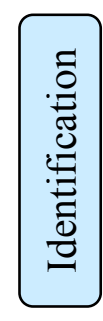

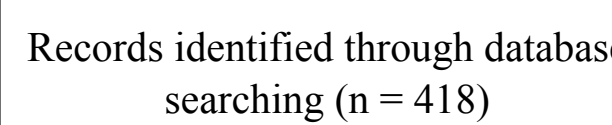
searching $(\mathrm{n}=418)$
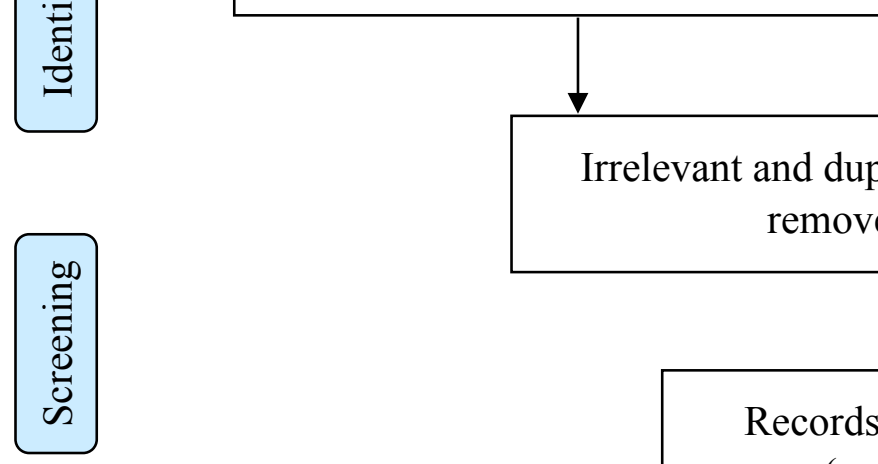

All records were systematically reviewed

$$
(\mathrm{n}=418)
$$

Irrelevant and duplicate records were removed (361)

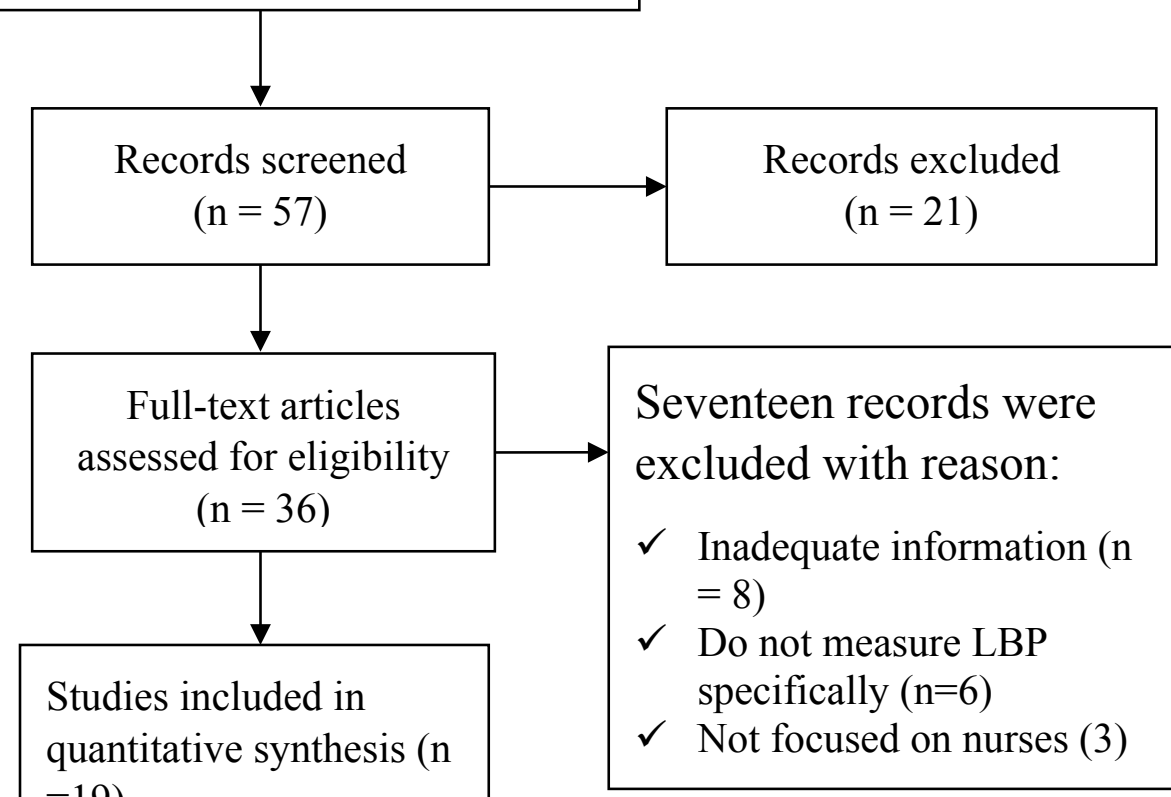

Figure 1: PRISMA flow diagram on prevalence of Low Back Pain (LBP) among nurses working at different healthcare facilities in Africa, 2018. 


\section{Critical appraisal result of the included studies}

A valid and standardized questionnaires were the main data collection tools in the included studies because of this, criterion number 8 and 9 in the selected critical appraisal instrument were not applicable for most studies except studies done by (55) as they utilized both interview and physical examination techniques to gather the data. Studies done by (31)(56)(57) utilized both selfadministered questionnaire and physical examination so that they utilized criterion number 9 as a critical appraisal item. All the included studies for this systematic review and meta-analysis were methodologically assessed and they satisfied the indicated criteria (Table 1). 
Table 1: Critical appraisal result of the included studies

\begin{tabular}{|c|c|c|c|c|c|c|c|c|c|c|c|c|c|}
\hline \multirow{2}{*}{ Included articles } & \multicolumn{13}{|c|}{ Criterion No. } \\
\hline & 1 & 2 & 3 & 4 & 5 & 6 & 7 & 8 & 9 & 10 & 11 & 12 & $\%$ \\
\hline Thembelihle D. et al(58) & $\checkmark$ & $\checkmark$ & $\checkmark$ & $\checkmark$ & $\checkmark$ & $\checkmark$ & $\checkmark$ & NA & NA & $x$ & $\checkmark$ & $\checkmark$ & 90 \\
\hline Asmare Y. et al.(55) & $\checkmark$ & $x$ & $\checkmark$ & $\checkmark$ & $\checkmark$ & $\checkmark$ & $\checkmark$ & $\checkmark$ & $\checkmark$ & $x$ & $\checkmark$ & $\checkmark$ & 83 \\
\hline M M. Belay et al.(59) & $\checkmark$ & $\checkmark$ & $\checkmark$ & $\checkmark$ & $\checkmark$ & $\checkmark$ & $\checkmark$ & NA & NA & $x$ & $\checkmark$ & $\checkmark$ & 90 \\
\hline Lamina S. et al.(21) & $\checkmark$ & $x$ & $\checkmark$ & $\checkmark$ & $\checkmark$ & $\checkmark$ & $\checkmark$ & NA & NA & $x$ & $\checkmark$ & $\checkmark$ & 80 \\
\hline Lamina S. et al.(21) & $\checkmark$ & $x$ & $\checkmark$ & $\checkmark$ & $\checkmark$ & $\checkmark$ & $\checkmark$ & NA & NA & $x$ & $\checkmark$ & $\checkmark$ & 80 \\
\hline F. O. Omokhodion et al.(60) & $\checkmark$ & $\checkmark$ & $\checkmark$ & $\checkmark$ & $\checkmark$ & $\checkmark$ & $\checkmark$ & NA & NA & $x$ & $\checkmark$ & $\checkmark$ & 90 \\
\hline Sikiru L \& Hanifa S(61) & $\checkmark$ & $\checkmark$ & $\checkmark$ & $\checkmark$ & $\checkmark$ & $\checkmark$ & $\checkmark$ & NA & NA & $\checkmark$ & $\checkmark$ & $\checkmark$ & 100 \\
\hline Muhammed A. et al(28) & $\checkmark$ & $x$ & $\checkmark$ & $\checkmark$ & $\checkmark$ & $\checkmark$ & $\checkmark$ & NA & NA & $x$ & $\checkmark$ & $\checkmark$ & 80 \\
\hline Mukaruzima Lela(62) & $\checkmark$ & $\checkmark$ & $\checkmark$ & $\checkmark$ & $\checkmark$ & $\checkmark$ & $\checkmark$ & NA & NA & $x$ & $\checkmark$ & $\checkmark$ & 90 \\
\hline Thembelihle D.(63) & $\checkmark$ & $x$ & $\checkmark$ & $\checkmark$ & $\checkmark$ & $\checkmark$ & $\checkmark$ & NA & NA & $x$ & $\checkmark$ & $\checkmark$ & 80 \\
\hline Chandeu Mwilila(64) & $\checkmark$ & $\checkmark$ & $\checkmark$ & $\checkmark$ & $\checkmark$ & $\checkmark$ & $\checkmark$ & NA & NA & $\checkmark$ & $\checkmark$ & $\checkmark$ & 100 \\
\hline Wided B. et al(31) & $\checkmark$ & $x$ & $\checkmark$ & $\checkmark$ & $\checkmark$ & $\checkmark$ & $\checkmark$ & NA & $\checkmark$ & $x$ & $\checkmark$ & $\checkmark$ & 82 \\
\hline Ian G Munabi. et al.(65) & $\checkmark$ & $x$ & $\checkmark$ & $\checkmark$ & $\checkmark$ & $\checkmark$ & $\checkmark$ & NA & NA & $x$ & $\checkmark$ & $\checkmark$ & 80 \\
\hline Mengestie M. et al.(59) & $\checkmark$ & $x$ & $\checkmark$ & $\checkmark$ & $\checkmark$ & $\checkmark$ & $\checkmark$ & NA & NA & $x$ & $\checkmark$ & $\checkmark$ & 80 \\
\hline Betty C.(26) & $\checkmark$ & $x$ & $\checkmark$ & $\checkmark$ & $\checkmark$ & $\checkmark$ & $\checkmark$ & NA & NA & $x$ & $\checkmark$ & $\checkmark$ & 80 \\
\hline Amany M. et al.(56) & $x$ & $x$ & $\checkmark$ & $\checkmark$ & $\checkmark$ & $\checkmark$ & $\checkmark$ & NA & $\checkmark$ & $\checkmark$ & $\checkmark$ & $\checkmark$ & 82 \\
\hline Ziadi B. et al.(66) & $\checkmark$ & $x$ & $\checkmark$ & $\checkmark$ & $\checkmark$ & $\checkmark$ & $\checkmark$ & NA & NA & $x$ & $\checkmark$ & $\checkmark$ & 80 \\
\hline Bolanle MS. et al.(57) & $\checkmark$ & $\checkmark$ & $\checkmark$ & $\checkmark$ & $\checkmark$ & $\checkmark$ & $\checkmark$ & NA & $\checkmark$ & $x$ & $\checkmark$ & $\checkmark$ & 91 \\
\hline Chiwaridzo et al.(67) & $\checkmark$ & $x$ & $\checkmark$ & $\checkmark$ & $\checkmark$ & $\checkmark$ & $\checkmark$ & NA & NA & $x$ & $\checkmark$ & $\checkmark$ & 80 \\
\hline
\end{tabular}


Based on the exclusion and inclusion criteria, 19 studies $((26,28,31,38,44,55,58,61,60,62-$ $65,59,56,66,57,67)$ ) were included in the final analysis. All studies were done using a cross sectional study design and within the included 19 studies, a total of 6110 nurses participated. Regarding the study participants, most of them were females even if some studies $(21,28,31,66)$ failed to report number of male and female participants in their studies clearly. Whereas a study done by (56) only incorporated female participants in their study. The sample size of the studies ranged between 80 which was a study done in Nigeria (60) and 880 a study conducted in Uganda (65). Concerning to the study settings, almost all were conducted in a hospital basis except one study which was done in Ethiopia, in addition to hospital nurses investigators incorporated nurses from health centers (55) (Table 2). 
Table 2: Characteristics of included articles in the systematic review and meta-analysis, 2018.

\begin{tabular}{|c|c|c|c|c|c|c|c|c|c|c|c|c|c|}
\hline Authors name & $\begin{array}{r}\text { Year of } \\
\text { publica } \\
\text { tion }\end{array}$ & Country & $\begin{array}{r}\text { Region } \\
\text { in the } \\
\text { continen } \\
\mathrm{t}\end{array}$ & Setting & $\begin{array}{l}\text { Study } \\
\text { design }\end{array}$ & $\begin{array}{r}\text { Sample } \\
\text { size }\end{array}$ & $\begin{array}{r}\text { Sampling } \\
\text { method }\end{array}$ & $\begin{array}{l}\text { Measur } \\
\text {-ement }\end{array}$ & $\begin{array}{r}\text { Gender } \\
(\%)\end{array}$ & $\begin{array}{r}\text { Mean } \\
\text { age }\end{array}$ & $\begin{array}{r}\text { Response } \\
\text { rate (\%) }\end{array}$ & $\begin{array}{l}\text { No. of } \\
\text { people } \\
\text { with the } \\
\text { outcome }\end{array}$ & $\begin{array}{c}\text { Preval- } \\
\text { ence } \\
(\%)\end{array}$ \\
\hline Thembelihle D. et al & 2018 & $\begin{array}{l}\text { South } \\
\text { Africa }\end{array}$ & South & $\begin{array}{l}\text { Regional } \\
\text { Hospital }\end{array}$ & $\begin{array}{l}\text { cross- } \\
\text { sectio } \\
\text { nal }\end{array}$ & 373 & $\begin{array}{l}\text { Convenie } \\
\text { nce }\end{array}$ & SSAQ & $\begin{array}{l}89 \% \\
\text { Female }\end{array}$ & ND & 65.0 & 157 & 59 \\
\hline Asmare Y. et al. & 2015 & Ethiopia & East & $\begin{array}{l}\text { Public } \\
\text { Hospital \& HC }\end{array}$ & $\begin{array}{l}\text { cross- } \\
\text { sectio } \\
\text { nal }\end{array}$ & 428 & Survey & $\begin{array}{l}\text { A- } \\
\text { NMSQ }\end{array}$ & $\begin{array}{l}53.7 \% \\
\text { Female }\end{array}$ & 30 & $91 \%$ & 222 & 57.1 \\
\hline M M. Belay et al. & 2016 & Ethiopia & East & $\begin{array}{l}\text { Public } \\
\text { Hospital }\end{array}$ & $\begin{array}{l}\text { cross- } \\
\text { sectio } \\
\text { nal }\end{array}$ & 430 & SRS & $\begin{array}{l}\text { SSAQ + } \\
\text { VAS }\end{array}$ & $\begin{array}{l}72.2 \% \\
\text { Female }\end{array}$ & 30.6 & $92 \%$ & 181 & 45.8 \\
\hline Lamina S. et al. & 2009 & Ethiopia & East & $\begin{array}{l}\text { Public } \\
\text { Hospital }\end{array}$ & $\begin{array}{l}\text { cross- } \\
\text { sectio } \\
\text { nal }\end{array}$ & 120 & $\begin{array}{l}\text { Convenie } \\
\text { nce }\end{array}$ & SSAQ & ND & ND & $83 \%$ & 60 & 60 \\
\hline Lamina S. et al. & 2009 & Nigeria & West & $\begin{array}{l}\text { Specialized } \\
\text { hospital }\end{array}$ & $\begin{array}{l}\text { cross- } \\
\text { sectio } \\
\text { nal }\end{array}$ & 500 & $\begin{array}{l}\text { Convenie } \\
\text { nce }\end{array}$ & SSAQ & ND & ND & $82 \%$ & 300 & 73.5 \\
\hline F. O. Omokhodion et al. & 2000 & Nigeria & West & Rural hospital & $\begin{array}{l}\text { cross- } \\
\text { sectio } \\
\text { nal }\end{array}$ & 80 & $\begin{array}{l}\text { Convenie } \\
\text { nce }\end{array}$ & SSAQ & $\begin{array}{l}33.8 \% \\
\text { Female }\end{array}$ & 43.8 & $93 \%$ & 51 & 69 \\
\hline Sikiru L \& Hanifa S & 2010 & Nigeria & West & $\begin{array}{l}\text { Specialized } \\
\text { hospital }\end{array}$ & $\begin{array}{l}\text { cross- } \\
\text { sectio } \\
\text { nal }\end{array}$ & 500 & ND & SSAQ & $\begin{array}{l}63.7 \% \\
\text { Female }\end{array}$ & 39.2 & $82 \%$ & 300 & 73.5 \\
\hline Muhammed A. et al & 2015 & Nigeria & West & UTH & $\begin{array}{l}\text { cross- } \\
\text { sectio } \\
\text { nal }\end{array}$ & 120 & $\begin{array}{l}\text { multi- } \\
\text { stage }\end{array}$ & SSAQ & ND & ND & $82 \%$ & 81 & 82.7 \\
\hline Mukaruzima Lela & 2010 & Rwanda & East & $\begin{array}{l}\text { Military } \\
\text { Hospital }\end{array}$ & $\begin{array}{l}\text { cross- } \\
\text { sectio } \\
\text { nal }\end{array}$ & 133 & SRS & $\begin{array}{l}\text { IPAQ+ } \\
\text { NMDQ }\end{array}$ & $\begin{array}{l}82 \% \\
\text { Female }\end{array}$ & 34.5 & $82 \%$ & 88 & 78 \\
\hline
\end{tabular}




\begin{tabular}{|c|c|c|c|c|c|c|c|c|c|c|c|c|c|}
\hline Thembelihle D. & 2010 & $\begin{array}{l}\text { South } \\
\text { Africa }\end{array}$ & South & $\begin{array}{l}\text { Public } \\
\text { Hospital }\end{array}$ & $\begin{array}{l}\text { cross } \\
\text { sectio } \\
\text { nal }\end{array}$ & 373 & SRS & SSAQ & $\begin{array}{l}79.3 \% \mathrm{~F} \\
\text { emale }\end{array}$ & ND & $72 \%$ & 158 & 59 \\
\hline Chandeu Mwilila & 2008 & $\begin{array}{l}\text { Tanzani } \\
\text { a }\end{array}$ & East & $\mathrm{MOI}$ & $\begin{array}{l}\text { cross } \\
\text { sectio } \\
\text { nal }\end{array}$ & 312 & $\begin{array}{l}\text { Purposiv } \\
\text { e }\end{array}$ & SSAQ & $\begin{array}{l}83.6 \% \mathrm{~F} \\
\text { emale }\end{array}$ & 35.9 & $54 \%$ & 126 & 73.6 \\
\hline Wided B. et al & 2017 & Tunisia & North & $\begin{array}{l}\text { Teaching } \\
\text { hospital }\end{array}$ & $\begin{array}{l}\text { cross- } \\
\text { sectio } \\
\text { nal }\end{array}$ & 329 & ND & $\begin{array}{l}\text { Borg } \\
\text { CR-10 } \\
\text { scale, } \\
\text { JCQ }\end{array}$ & ND & 39.8 & $61.70 \%$ & 125 & 58.1 \\
\hline Ian G Munabi. et al. & 2014 & Uganda & East & $\begin{array}{l}\text { Public } \\
\text { Hospital }\end{array}$ & $\begin{array}{l}\text { cross- } \\
\text { sectio } \\
\text { nal }\end{array}$ & 880 & ND & $\begin{array}{l}\text { DMQ \& } \\
\text { NMDQ }\end{array}$ & $\begin{array}{l}85.7 \% \mathrm{~F} \\
\text { emale }\end{array}$ & 35.4 & $85.40 \%$ & 459 & 61.9 \\
\hline Mengestie M. et al. & 2016 & Ethiopia & East & $\begin{array}{l}\text { Public } \\
\text { Hospital }\end{array}$ & $\begin{array}{l}\text { cross- } \\
\text { sectio } \\
\text { nal }\end{array}$ & 395 & SRS & SSAQ & $\begin{array}{l}72.2 \% \mathrm{~F} \\
\text { emale }\end{array}$ & 30.6 & 91.9 & 166 & 45.8 \\
\hline Betty C. & 2015 & Kenya & East & $\begin{array}{l}\text { Both public \& } \\
\text { private } \\
\text { hospitals }\end{array}$ & $\begin{array}{l}\text { cross- } \\
\text { sectio } \\
\text { nal }\end{array}$ & 169 & SRS & SSAQ & $\begin{array}{l}76.9 \% \mathrm{~F} \\
\text { emale }\end{array}$ & 35 & 76.9 & 100 & 76.9 \\
\hline Ziadi B. et al. & 2014 & Algeria & North & $\begin{array}{l}\text { Public } \\
\text { Hospital }\end{array}$ & $\begin{array}{l}\text { cross- } \\
\text { sectio } \\
\text { nal }\end{array}$ & 450 & SRS & SSAQ & ND & ND & 66.7 & 200 & 66.7 \\
\hline Bolanle MS. et al. & 2010 & Nigeria & West & $\mathrm{UH}, \mathrm{GH}, \mathrm{PH}$ & $\begin{array}{l}\text { cross- } \\
\text { sectio } \\
\text { nal }\end{array}$ & 160 & SRS & SSAQ & $\begin{array}{l}97.5 \% \mathrm{~F} \\
\text { emale }\end{array}$ & 36.4 & 80 & 56 & 44.1 \\
\hline Chiwaridzo et al. & 2018 & $\begin{array}{l}\text { Zimbab } \\
\text { we }\end{array}$ & East & $\begin{array}{l}\text { Public } \\
\text { Hospital }\end{array}$ & $\begin{array}{l}\text { cross- } \\
\text { sectio } \\
\text { nal }\end{array}$ & 208 & $\begin{array}{l}\text { Stratified } \\
\text { RS }\end{array}$ & NMDQ & $\begin{array}{l}84.6 \% \mathrm{~F} \\
\text { emale }\end{array}$ & 32 & 55.7 & 65 & 55.7 \\
\hline
\end{tabular}




\section{Prevalence of low back pain (LBP)}

During the period 2000-2018, nineteen studies with a sample size of 6110 have been carried out. Among them, the lowest and the highest prevalence were found to be $44.1 \%$ and $82.7 \%$. Both the highest and the lowest prevalence of LBP were reported from a studies done in Nigeria. The lowest prevalence of LBP was reported by (57) whereas the highest prevalence was reported by (28). The estimation of the prevalence rate of LBP among nurses using the random effects model was found to be $64.07 \%$ (95\% CI: 58.68-69.46; P-value < 0.0001). Heterogeneity of the reviewed studies was $\mathrm{I}^{2}=94.2 \%$ and heterogeneity Chi-squared $=310.06(\mathrm{~d} . \mathrm{f}=18)$, P-value $<0.0001$ (Figure 2).

Study ID ES $(95 \% \mathrm{Cl})$

Thembelihle D. et al (2018)

Asmare Y. et al. (2015)

M M. Belay et al. (2016)

Lamina S. et al. (2009)

Lamina S. et al. (2009)

F. O. Omokhodion et al. (2000)

Sikiru L \& Hanifa S (2010)

Muhammed A. et al (2015)

Mukaruzima Lela (2010)

Thembelihle D. (2010)

Chandeu Mwilila (2008)

Wided B. et al (2017)

Ian G Munabi. et al. (2014)

Mengestie M. et al. (2016)

Betty C. (2015)

Amany M. et al. (2014)

Ziadi B. et al. (2014)

Bolanle MS. et al. (2010)

Chiwaridzo et al. (2018)

Overall (I-squared $=88.1 \%, p=0.000$ )

NOTE: Weights are from random effects analysis
$59.00(51.01,66.99) 5.28$

$57.10(49.17,65.03) 5.29$

$45.80(38.30,53.30) 5.36$

$60.00(51.98,68.02) 5.28$

$73.50(65.08,81.92) 5.22$

$69.00(60.70,77.30) 5.24$

$73.50(65.08,81.92) 5.22$

$82.70(74.05,91.35) 5.18$

$78.00(69.46,86.54) 5.20$

$59.00(51.01,66.99) 5.28$

$73.60(65.17,82.03) 5.22$

$58.10(50.14,66.06) 5.29$

$61.90(53.81,69.99) 5.27$

$45.80(38.30,53.30) 5.36$

$76.90(68.39,85.41) 5.20$

$79.30(70.73,87.87) 5.19$

$66.70(58.47,74.93) 5.25$

$44.10(36.68,51.52) 5.37$

$55.70(47.82,63.58) 5.30$

$64.07(58.68,69.46) 100.00$ 
Figure 2: Forest plot of prevalence of low back pain among nurse in the African healthcare facilities, 2018.

\section{Subgroup analysis}

According to the subgroup analyses, the highest prevalence of LBP among nurses was reported from west African region with prevalence rates of $68.46 \%$ (95\% CI: $54.94-81.97$; Pvalue $<0.0001)$ and followed by north Africa region with prevalence rate of $67.95 \%(95 \% \mathrm{CI}$ : 55.96-79.94; $\mathrm{P}$ value $<0.0001)$ had the higher prevalence of LBP as compared to their south African counterparts, 59\% (95\% CI: 51-66.9; P-value <0.0001) (Figure 3).

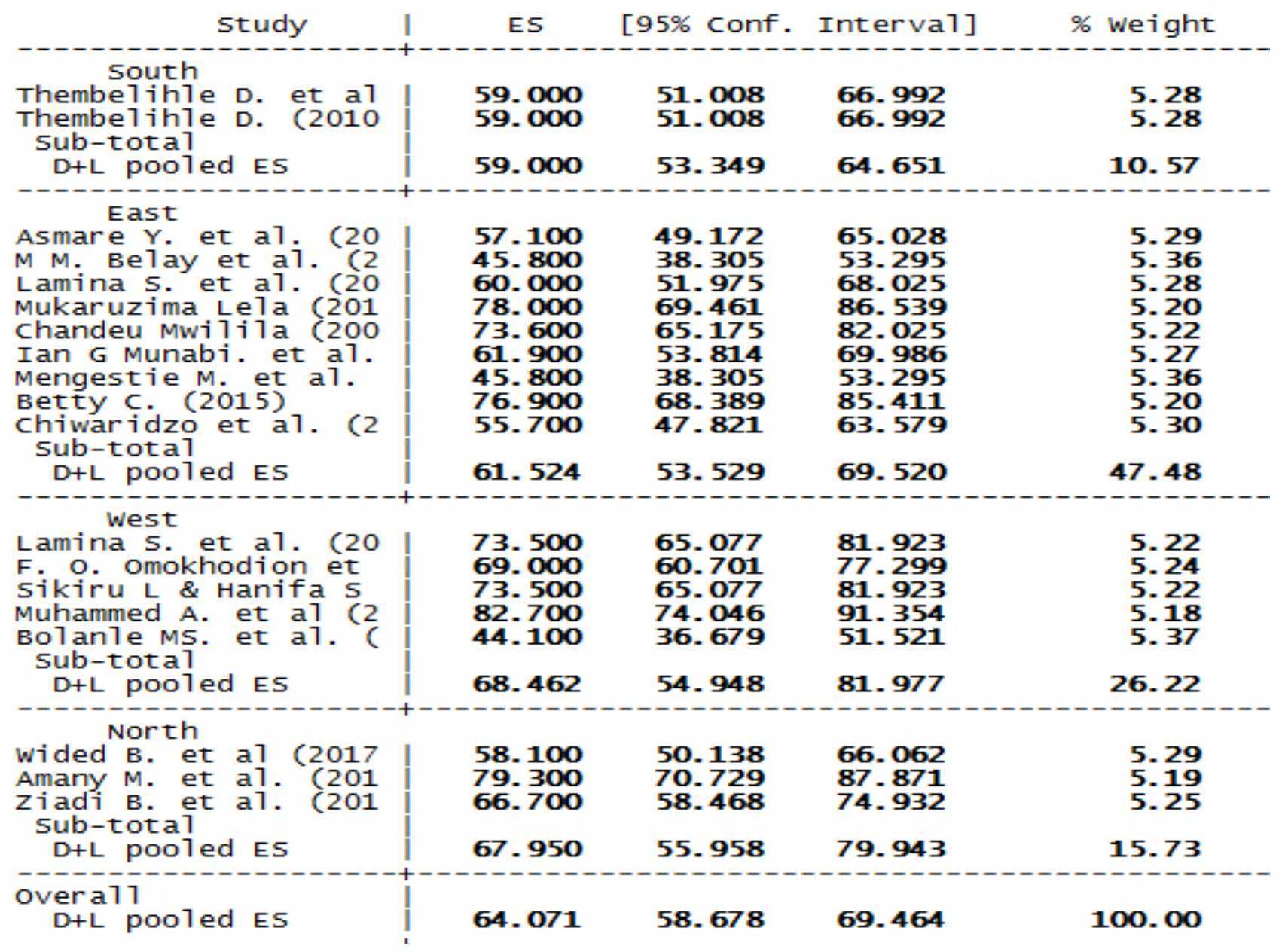

Figure 3: Subgroup analysis of low back pain among nurse using region of the continent in the African healthcare facilities, 2018. 
bioRxiv preprint doi: https://doi.org/10.1101/507053; this version posted December 27, 2018. The copyright holder for this preprint (which was not certified by peer review) is the author/funder, who has granted bioRxiv a license to display the preprint in perpetuity. It is made available under aCC-BY 4.0 International license. 


\section{Meta-regression}

Meta-regression analysis showed that there was no significant statistical relationship between the year of publication and the prevalence of the LBP $(\beta=-0.82$, P-value $=0.808)$ (Figure 4).

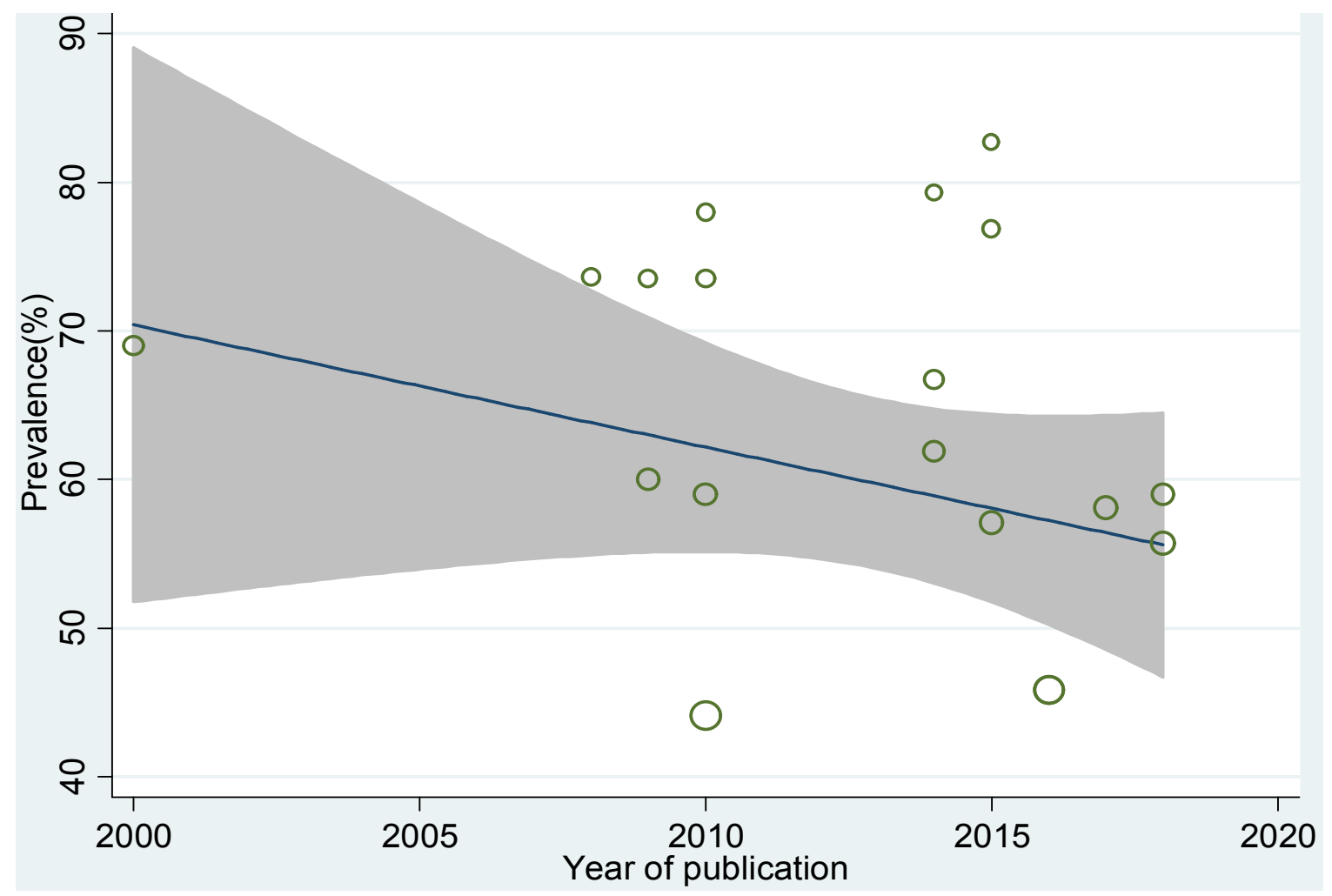

Figure 4: Funnel plot showing the relation between year of publication and prevalence of LBP among nurses working in different African health facilities, 2018.

The meta-regression also showed that there was no significant statistical relationship between the sample size and the prevalence of the LBP $(\beta=-0.007$, P-value $=0.93)$ (Figure 5). 


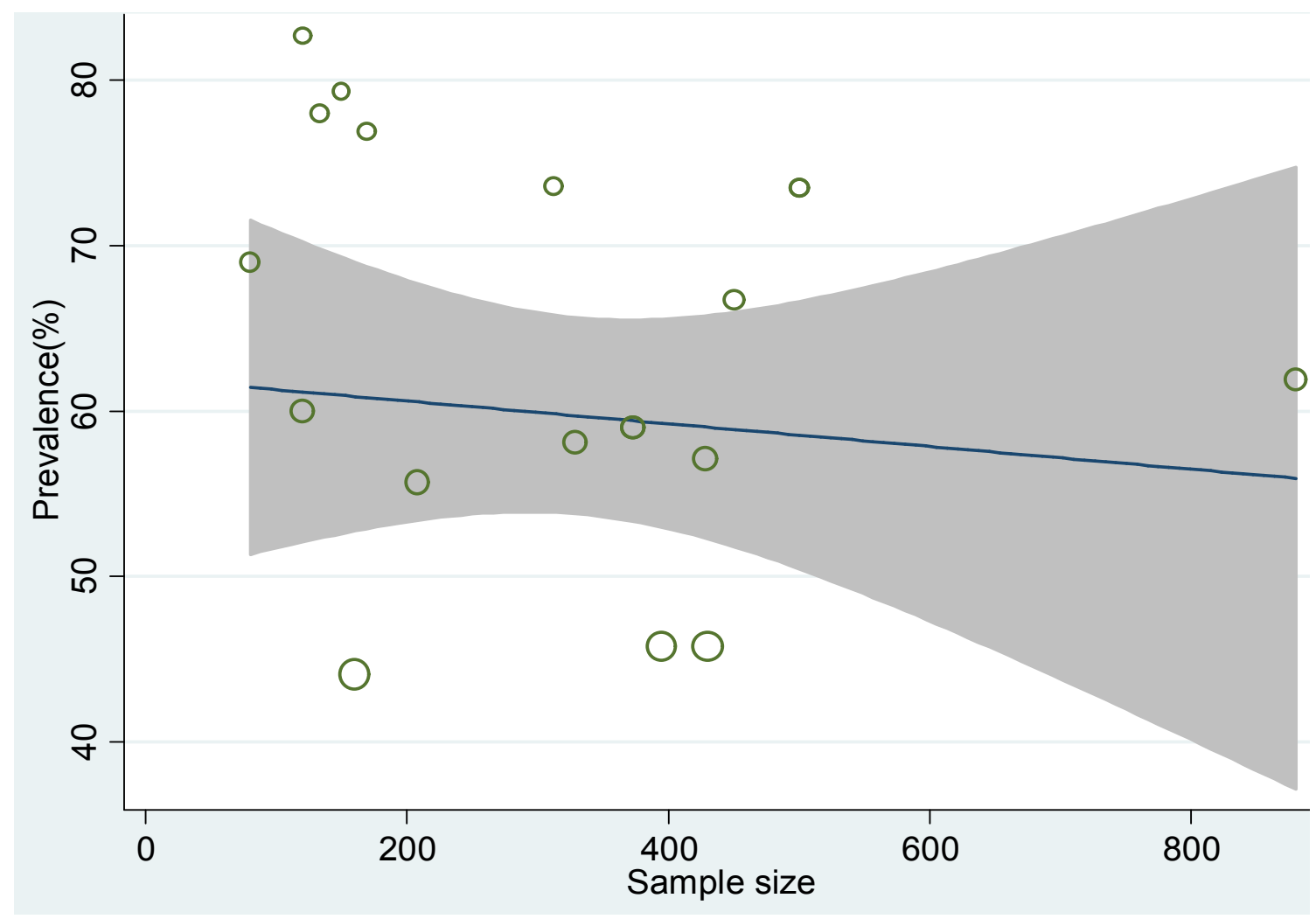

Figure 5: Funnel plot showing the relation between sample size and prevalence of LBP among nurses working in different African health facilities, 2018.

To assess publication bias, the funnel plot and the Egger's test was conducted in the metaanalysis. The funnel plot and Egger's regression tests $(\beta=-0.0024, \mathrm{SE}=0.06, \mathrm{P}=0.96)$ showed that no evidence of publication bias for the included studies (Figure 6). 


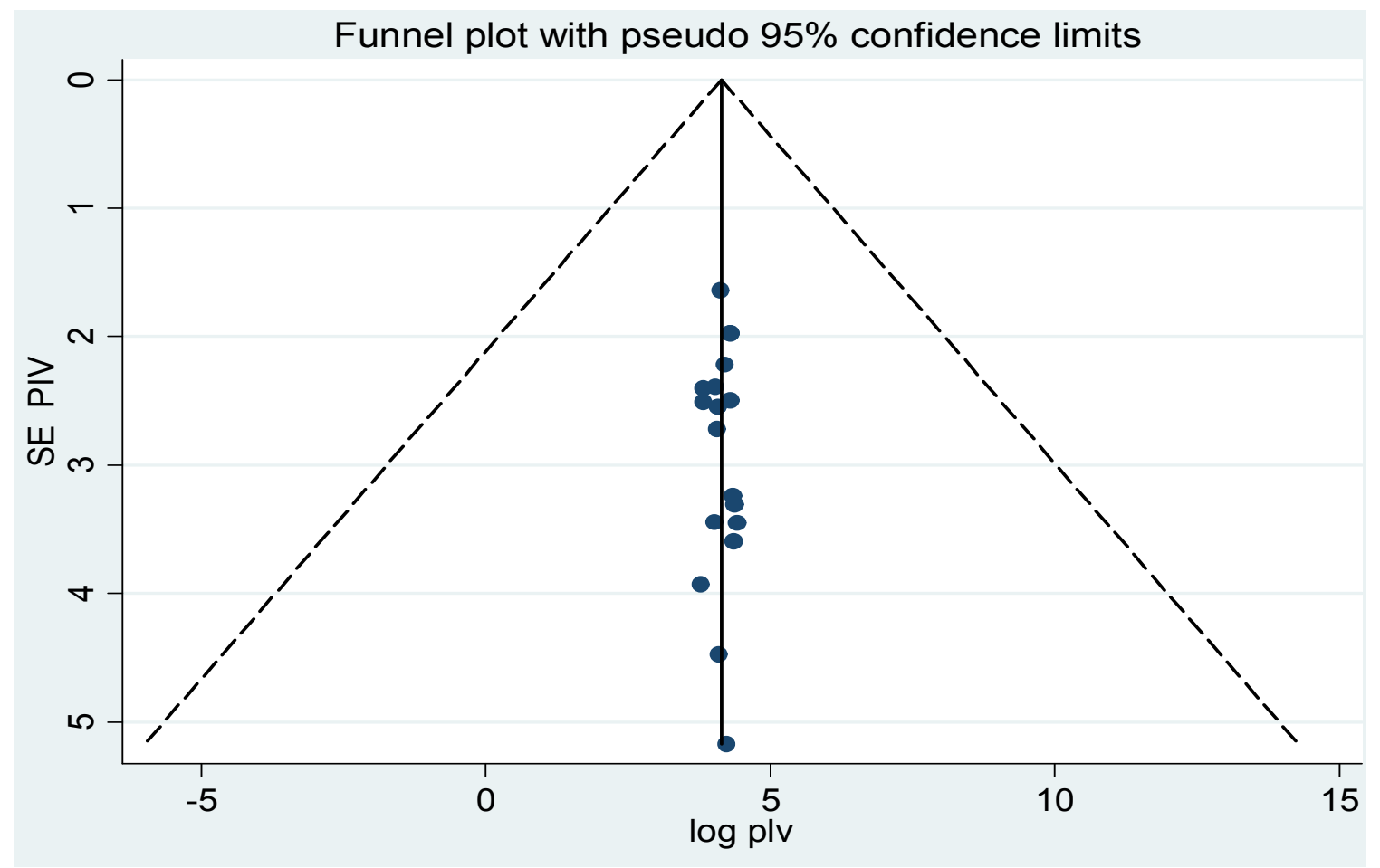

Figure 6: Funnel plot for assessing publication bias among studies, 2018 


\section{Discussion}

Low back pain is a common work- related musculoskeletal disorders in healthcare workers, with particularly imposing high risk on nursing professionals across different healthcare facilities mainly in hospital settings $(68,69)$. Such problems are reported to considerably influence on quality of life of healthcare professionals and this will in turn affects the healthcare quality (29).

This study denotes the first effort to report on the prevalence of LBP among nurses working in healthcare facilities in the African continent. The aim of this systematic review and metaanalysis was to determine the pooled prevalence of LBP among nurse working at different healthcare facilities in different African regions. By providing a comprehensive picture, this study would help to recognize the impact of the problem on nurse professionals in African countries.

Low Back Pain is a regular occupational problem for nurses worldwide, and has been previously reported at rates between $45 \%$ in England (70), 63\% in Australia (71). Research from Hong Kong and China has also showed that LBP may affect between 40.6\% (72) and 56\% (73) respectively. African studies report LBP rates between $44.1 \%, 79.4 \%$ and $82.7 \%(28,43,57,74)$

The result of the present systematic review and meta-analysis carried on professional nurses working at different regions of African healthcare facilities showed that the overall prevalence of LBP among nurses was $64.07 \%$. This finding was higher than a study done in Zimbabwe (67), Nigeria (57), Tunisia (10) and Iran (49) showed that the overall prevalence of LBP among nurses was $55.67 \%, 44.1 \%, 51.1 \%$ and $61.2 \%$ respectively.

Whereas a significant amount of studies conducted in the western nations and Asian countries revealed that the overall prevalence of LBP among nurses was higher when we compared to the present finding. A study done in Japan (75), Turkey (13) and United States of America (76) 
showed that the overall prevalence of LBP among nurses was $91.9 \%, 77.1 \%$ and $72.5 \%$ respectively which all indicated that the existence of more sever prevalence of LBP compared to the present study . Another study done in Swiss (77) and Italy (78) also revealed that the overall annual prevalence of LBP among nurses was found to be $73-76 \%$ and $86 \%$ respectively. This also confirms that there is higher prevalence of LBP among nurse in the western nations.

Studies done in the western and in some Asian countries revealed that the existence of higher prevalence of LBP among nurses. This finding is confirmed by different literatures in the subject area. This high existence of LBP among nurses in the developing nations might be high workloads (79) for patient care, conducting advanced procedures in different advanced area of patient care and this all might lead them to the experiencing of LBP in their working environment.

The results of this study identified the presence of high prevalence of low back pain among nurses who were working in the western region of Africa. In the present study, five different studies were incorporated from the western region of Africa and all of them were from Nigeria. Nigeria is the number one populous country in Africa. This has its own impact in the healthcare system including to the healthcare professionals. As it was mentioned in many literatures, nurses are the number one frontline healthcare professionals that can contact clients. This would have its own share to the development of LBP on nursing staffs.

\section{Limitation}

Adequate studies were not incorporated from some region of the continent and even most of the studies were concentrated in a single country in each region of the continent. This might have its own shortfalls in producing the overall picture of the problem to the continent as a whole. 


\section{Conclusion}

Even though the overall prevalence of the present study is lower when compared to the western and Asian studies, it indicated that the prevalence of low back pain among nurses is on the move.

Conducting a study at national levels in order to determine the physical, mental, supervisor support, nurse colleague interaction, psychological, and work setting stressors in the work environment of nurses and their relationship with low back pain should investigated. This would enhance on how to identify the risk factors and to design a detailed plans for the prevention and control of low back pain. All the efforts made would improve nurses' sense of belongingness, retention, quality of patient care and even organizational culture.

\section{Declarations}

\section{Availability of data and material:}

All data generated or analyzed during this study are included in this manuscript.

\section{Competing Interests:}

The authors declared that there is no competing interest.

\section{Funding:}

There was no funding received from any agent.

\section{Authors' Contributions}

AS was involved in the design of the study, data analysis, and interpretation of the findings, report writing, and paper preparation. YW \& EA involved in the analysis and interpretation of the data, and review of the report. WA approved the manuscript and all authors read and approved the final paper. All authors contributed equally to this work 


\section{Reference}

1. Cunningham C, Flynn T BC. Low back pain and occupation among Irish health workers. Occup Med. 2006;56(7):23-8.

2. Kamper, S. J. et al. Multidisciplinary biopsychosocial rehabilitation for chronic low back pain: Cochrane systematic review and meta-analysis. Br Med J. 2015;350:444.

3. Mafuyai MY, Babangida BG, Mador ES, Bakwa DD JY (2014). The increasing cases of lower back pain in developed Nations: a reciprocal effect of development. AJIS. 2014;3(5):23-8.

4. Hasan M. Keriri. Prevalence and risk factors of low back pain among nurses in operating rooms, Taif, Saudi Arabia. Am J Res Commun. 2013;1(11):45-70.

5. MacDonald, D., Moseley, L.G. \& Hodges WP (2008). Why do some patients keep hurting their back: Evidence of ongoing muscle dysfunction during remission from recurrent back pain? J Pain. 2008;142:183-7.

6. Brennan, G., Shafat, A., Mac Donncha, C. \& Vekins C. Lower back pain in physically demanding college academic programs: a questionnaire based study. Bio-Medical Cent Musculoskelet Disord 13,. 2007;13:67-75.

7. Burdorf, A. \& Jansen JP. Predicting the long term course of low back pain and its consequences for sickness absence and associated work disability. Occup Environ Med. 2005;63:522-9.

8. Vos T. et al. Years lived with disability (YLDs) for 1160 sequelae of 289 diseases and injuries 1990-20100: a systematic analysis for the Global Burden of Disease Study. Lancet [Internet]. 2012;380(9859):2163-96. Available from: http://dx.doi.org/10.1016/s01406736

9. Lipscomb J, Trinkoff A BB\& G-BJ. Health care system changes and reported musculoskeletal disorders among registered nurses. Am J Public Health. 2004;94:1431-5.

10. Bejia I, Younes M, Hadj J, Khalfallah T, Ben K. Prevalence and factors associated to low back pain among hospital staff. Rev du Rhum 72 [Internet]. 2004;72:427-32. Available 
from: 10.1016/j.rhum.2004.06.007

11. Wong TS, Teo N KM. Prevalence and risk factors associated with low back pain among healthcare providers in a district hospital. Malays Orthop J. 2010;4(2):23-8.

12. Johnson OE EE. Prevalence and risk factors of low back pain among workers in a health facility in South-South Nigeria. BJMMR 2016; 2016;11(8):1-8.

13. Karahan A, Kav S, Abbasoglu A, Dogan N. Low back pain: prevalence and associated risk factors among hospital staff. JAN [Internet]. 2009;65:516-24. Available from: https://doi.org/10.1111/j.1365-2648.2008.04905.x

14. Burdorf A SG. Positive and negative evidence of risk factors for back disorders. Scand J Work Env Heal. 1997;23(4):243-56.

15. Munjanja O, Kibuka S DD. The Nursing Workforce in Sub-Saharan Africa.InThe Global Nursing Review Initiative.Geneva. Int Counc Nurses. 2005;1-68.

16. Nelson A et al. Development and evaluation of a multifaceted ergonomics program to prevent injuries associated with patient handling tasks. Int J Nurs. 2006;43:717-33.

17. Hilkka Hllhlrnakl. Low-back pain, its origin and risk indicators. Scand J Work Env Heal. 1991;17:81-90.

18. Dlungwane T, Voce A, Knight S. Prevalence and factors associated with low back pain among nurses at a regional hospital in KwaZulu-Natal, South Africa. Heal SA Gesondheid [Internet]. 2018;1:2071. Available from: http://www.scielo.org.za/pdf/hsa/v23/04.pdf

19. Alexandre, N.M.C., Angerami, E.L.S. and Moreira Filho D. Back Pain and Nursing. Rev da Esc Enferm da USP. 1996;30:267-85.

20. Salvi Shah BD. Prevalence of Low Back Pain and Its Associated Risk Factors among Doctors in Surat. Int J Heal Sci Res. 2012;2(1):1-5.

21. Sikiru L, Shmaila H. Prevalence and Risk Factors of Low Back Pain among Nurses in Africa: Nigerian and Ethiopian Specialized Hospitals Survey Study. East African J Pablic Heal. 2009;6(1):22-6.

22. Cesana G, Arduca A, Latocca R and SG. Risk evaluation and health surveillance in 
hospitals: A critical review and contribution regarding experience obtained at Garardo dei Tintori Hospital in Monza. Med. Med -Lav 1998; 1998;89(1):23-46.

23. Vieira, E.R., Kumar, S., Coury, H. J.C.G. \& Narayan Y. Low back problems and possible improvements in nursing jobs. J Adv Nurs. 2006;55(1):79-89.

24. Triolo PK. Occupational health hazard of hospital staff nurses. Part II Physical, chemical and biological stressors. AAOHN - J. 1988;37(7):274-9.

25. Gourmelen J et al. Frequency of Low Back Pain among Men and Women Aged 30 to 64 Years in France. Results of Two National Surveys. Ann Réadaptation Médecine Phys [Internet]. 2007;50:640-4. Available from: https://doi.org/10.1016/j.annrmp.2007.05.009

26. Betty Chebet Tanui. Assessment of Work-Related Musculoskeletal Disorders among Nurses in Mombasa County, Kenya. 2015.

27. Tate, R.B., Yassi, A. \& Cooper J. Predictorsof Time Loss after Back Injury in Nurses. Spine (Phila Pa 1976). 1999;24:1930-5.

28. Farooq MA, Awwal LM, Musa HA, Mustapha GA. Work-Related Risk Factors for Lower Back Pain Among Nurses In Ahmadu Bello University Teaching Hospital ( ABUTH ), Zaria-Nigeria. IOSR J Nurs Heal Sci. 2015;4(3):20-5.

29. Punnett, L. and Wegman D. Work-Related Musculoskeletal Disorders: The Epidemiologic Evidence and the Debate. J Electromyogr Kinesiol [Internet]. 2004;14:13-23. Available from: https://doi.org/10.1016/j.jelekin.2003.09.015

30. De Castro A. Handle with Care: The American Nurses Association's Campaign to Address Work-Related Musculoskeletal Disorders. Online J Issues Nurs. 2004;9(3):10-2.

31. Boughattas W, Maalel O El, Maoua M, Bougmiza I. Low Back Pain among Nurses : Prevalence, and Occupational Risk Factors. Occup Dis Environ Med [Internet]. 2017;5:2637. Available from: https://doi.org/10.1016/j.annrmp.2007.05.009

32. Smith, D. R., Wei, N, Kang, L., Wang RS. Musculoskeletal disorders among professional nurses in mainland China. J Prof Nurs. 2004;20(6):390-5.

33. Holder, N. et al. Cause, prevalence, and response to occupational musculoskeletal injuries 
reported by physical therapists and physical therapist assistants. Phys Ther. 2009;79(7):4552.

34. Asadi P, Monsef Kasmaei V, Zia Ziabari S ZB. The prevalence of low back pain among nurses working in Poursina hospital in Rasht, Iran. J Emerg Pr Trauma [Internet]. 2016;2(1):11-5. Available from: doi: 10.15171/jept.2015.01.

35. Shogren E. The relationship between the nursing shortage and nursing injury. In Back Injury among Health Care Workers, Lewis Publ. 2004;247-52.

36. Collins JW \& Menzel. Scope of the problem. InSafe Patient Handling and Movement: A Practical Guide for Health Care Professionals. Springer. 2006;8-9.

37. Charney W \& Schirmer J (2007). Nursing injury rates and negative patient outcomesconnecting the dots. AAOHN J. 2007;55:470-5.

38. Sikiru L, Hanifa S. Prevalence and risk factors of low back pain among nurses in a typical Nigerian hospital. Afr Health Sci. 2010;10(1).

39. Julie M. Fritz SNC. Low Back Pain in Adolescents: A Comparison of Clinical Outcomes in Sports Participants and Nonparticipants. J Athl Train. 2010;45(1):61-6.

40. Bos E, Krol B, van der Star L GJ (2007). Risk factors and musculoskeletal complaints in non-specialized nurses, IC nurses, operation room nurses, and X-ray technologists. Int Arch Occup Env Heal. 2007;80:198-206.

41. Ando S . et al. Associations of self-estimated workloads with musculoskeletal symptoms among hospital nurses. Occup. Occup Env Med. 2000;57(211-216):2000.

42. Costa BR VE (2010). Risk factors for work-related musculoskeletal disorders: A systematic review of recent longitudinal studies. Am J Ind Med. 2010;53:285-323.

43. TinubuBM, Mbada CE, Oyeyemi AL FA. Work-related musculoskeletal disorders among nurses in Ibadan, South-west Nigeria: a cross-sectional survey. BMC Musculoskelet Disord. 2010;11:12.

44. M M. Belay, A Worku, S A. Gebrie BLW. Epidemiology of Low Back Pain among Nurses Working in Public Hospitals of Addis Ababa, Ethiopia. East Cent African J Surg. 
2016;21(1):113-31.

45. Alexopoulos EC, Burdorf A KA. Risk factors for musculoskeletal disorders among nursing personnel in Greek hospitals. Int Arch Occup Env Heal. 2003;76(4):289-94.

46. Hou JY SJ. Risk factors for musculoskeletal discomfort in nurses. J Nurs Res. 2006;14:22836.

47. Sikiru L HS (2010). Prevalence and risk factors of low back pain among nurses in a typical Nigerian hospital. Afr Heal Sci 10. 2010;10:26-30.

48. Moher D, Liberati A, Tetzlaff J, Altman DG PG. Preferred reporting items for systematic reviews and meta-analyses: the PRISMA statement. PLoS Med [Internet]. 2009;6(6). Available from: doi: 10.1371/journal.pmed 1000097.

49. Azizpour Y, Delpisheh A, Montazeri Z, Sayehmiri K. Prevalence of low back pain in Iranian nurses: a systematic review and meta- analysis. BMC Nurs [Internet]. 2017;16(50):1-10. Available from: 10.1186/s12912-017-0243-1

50. Chiou WK, Wong MK LY. Epidemiology of low back pain in Chinese nurses. Int J Nurs Stud. 1994;31(4):361-8.

51. Walker BF. The prevalence low back pain: a systematic review of the literature from 1966 to 1998. J Spinal Disord. 2000;13(3):205-2017.

52. Mousavi SJ et al. Low back pain in Iran: a growing need to adapt and implement evidencebased practice in developing countries. Spine (Phila Pa 1976) [Internet]. 2011;36(10). Available from: 10.1097/BRS.0b013e3181 fa1da2

53. Louw QA, Morris LD G-SK. The prevalence of low back pain in Africa: a systematic review. BMC Musculoskelet Disord [Internet]. 2007;8(105). Available from: 10. $1186 / 1471-2474-8-105$

54. Ades AE, Lu G HJ. The interpretation of random-effects metaanalysis in decision models. Med Decis Mak. 2005;25:646-54.

55. Institutions H, Yitayeh A, Mekonnen S, Fasika S, Gizachew M. Emergency Medicine : Open Access Annual Prevalence of Self-Reported Work Related Musculoskeletal Disorders 
and Associated Factors among Nurses Working at Gondar Town Governmental Health Institutions, Northwest Ethiopia. Emerg Med (Los Angel). 2015;5(1):1-7.

56. El-soud AMA, El-najjar AR, El-fattah NA, Hassan AA. Prevalence of low back pain in working nurses in Zagazig University Hospitals: an epidemiological study. Egypt Rheumatol Rehabil. 2014;41:109-15.

57. Tinubu BMS, Mbada CE, Oyeyemi AL, Fabunmi AA. Work-Related Musculoskeletal Disorders among Nurses in Ibadan, South-west Nigeria : a cross-sectional survey. BMC Musculoskelet Disord. 2010;11(12):6-13.

58. Dlungwane Thembelihle AV\& SK. Prevalence and factors associated with low back pain among nurses at a regional hospital in KwaZulu-Natal, South Africa. Heal SA Gesondheid [Internet]. 2014;1-6. Available from: http://www.hsag.org.za

59. Mengestie M. Belay, A Worku, SA Gebrie BW. Epidemiology of low back pain among nurses working in public hospitals of Addis Ababa, Ethiopia. East Cent African J Surg. 2016;21(1):139040.

60. Omokhodion et al. Prevalence of Low Back Pain among Staff in a Rural Hospital in Nigeria. Occup Med (Chic Ill) [Internet]. 2000;50(2):1396354. Available from: https:/academic.oup.com/occmed/article/50/2/107/1396354\%0AF.

61. Sikiru L, Shmaila H. Prevalence and risk factors of low back pain among nurses in Africa: Nigerian and Ethiopian specialized hospitals survey study. East African J Pablic Heal. 2009;6(1):22-6.

62. Mukaruzima Lela. The Relationship between Physical Activity and Low Back Pain among Nurses in Kanombe Military Hospital. University of the Western Cape; 2010.

63. Thembelihle. Prevalence of Low Back Pain Amongst Nurses at Edendale Hospital A dissertation submitted to the Department of Public Health Medicine Nelson R . Mandela School of Medicine Durban, South Africa In partial fulfilment of the requirements for the Master in Pu. University of KwaZulu-Natal; 2010.

64. Chandeu M. Work-Related Low Back Pain among Clinical Nurses in Tanzania. University of the Western Cape.; 2008. 
65. Munabi IG, Buwembo W, Kitara DL, Ochieng J, Mwaka ES. Musculoskeletal disorder risk factors among nursing professionals in low resource settings : a cross-sectional study in Uganda. BMC Nurs 2014,. 2014;13(7):1-8.

66. Boukerma Z, Behlouli AL, Reggad M. Epidemiology of low back pain among nurses of the hospital of Sétif (Algeria). BMJ Occopational Environ Med [Internet]. 2014;71(1):1-6. Available from: https://oem.bmj.com/content/71/Suppl_1/A113.3\%0A1.

67. Chiwaridzo M, Makotore V, Dambi JM, Munambah N, Mhlanga M. Work - related musculoskeletal disorders among registered general nurses : a case of a large central hospital in Harare , Zimbabwe. BMC Res Notes [Internet]. 2018;11(315):1-7. Available from: https://doi.org/10.1186/s13104-018-3412-8

68. Wilkinson WE, Salazar MK, Uhl JE, Koepsell TD, DeRoos RL LR. Occupational injuries: a study of health care workers at a northwestern health science center and teaching hospital. Am Assoc Occup Heal Nurs J. 1992;40:287-93.

69. Stubbs DA, Buckle P, Hudson MP, Butler PE RP. Back pain in the nursing profession, part I: epidemiology and pilot methodology. Ergon. 1983;26:755-65.

70. Smedley J, Egger P, Cooper C CD. Manual handling activities and risk of low back pain in nurses. Occup Env Med. 1995;52(3):160-3.

71. Lusted MJ, Carrasco CL, Mandryk JA HS. Self reported symptoms in the neck and upper limbs in nurses. Appl Ergon. 1996;27(6):381-7.

72. Yip YB: A. A study of work stress, patient handling activities and the risk of low back pain among nurses in Hong Kong. Aust J Adv Nurs 2001,. 2001;36:796-804.

73. Smith DR, Wei N, Kang L WR. Musculoskeletal disorders among professional nurses in mainland China. J Prof Nurs 2004,. 2004;20(6):390-5.

74. Fabunmi, A. A. And Gbiri CA. Relationship between balance performance in the elderly and some anthropometric variables. Afr J Med Med Sci. 2008;37(4):321-6.

75. Smith DR, Kondo N, Tanaka E, Tanaka H, Hirasawa K YZ. Musculoskeletal disorders among hospital nurses in rural Japan. Rural Remote Heal J. 2003;241(3):online. 
76. Josephson M, Largerstrom M, Hagberg M HE. Musculoskeletal symptoms and job strain among nursing personnel: a study over a three year period. Occup Env Med. 1997;52:4757.

77. Maul I, Läubli T, Klipstein A KH. Course of low back pain among nurses: a longitudinal study across eight years. Occup Env Med [Internet]. 2003;60(7):497. Available from: doi: 10.1136/oem.60.7.497

78. Corona G, Amedei F, Miselli F, Padalino MP, Tibaldi S FG. Association between relational and organizational factors and occurrence of musculoskeletal disease in health personnel. G Ital Med Lav Ergon [Internet]. 2005;27(2):208-2012. Available from: doi: 10.1136/oem.60.7.497

79. Shieh SH, Sung FC, Su CH, Tsai Y HV. Increased low back pain risk in nurses with high workload for patient care: a questionnaire survey. Taiwan J Obs Gynecol [Internet]. 2016;55(4):525-9. Available from: doi: 10.1016/j.tjog.2016.06.013 


\section{Appendix}

The critical appraisal tool utilized to assess the quality of the included studies.

Part I Is the final sample representative of the target population?

1. At least one of the following must apply in the study: an entire target population, randomly selected sample, or sample stated to represent the target population.

2. At least one of the following: reasons for non-response described,non responders described, comparison of responders and non-responders, or comparison of sample and target population

3. Response rate and, if applicable, drop-out rate reported

Part II Quality of the data?

4. Were the data primary data of low back pain or was it taken from a survey not specifically designed for that purpose?

5. Were the data collected from each adult directly or were they collected from a proxy?

6. Was the same mode of data collection used for all subjects?

7. At least one of the following in case of questionnaire: a validated questionnaire or at least tested for reproducibility.

8. At least one of the following in the case of an interview: Interview validated, tested for reproducibility, or adequately described and standardized.

9. At least one of the following in the case of an examination: Examination validated, tested for reproducibility, or adequately described and standardized.

Part III Definition of low back pain (LBP)

10. Was there a precise anatomic delineation of the lumbar area or reference to an easily obtainable article that contains such specification?

11. Was there further useful specification of the definition of LBP, or question(s) put to study subjects quoted such as the frequency, duration or intensity, and character of the pain. Or was there reference to an easily obtainable article that contains such specification?

12. Were recall periods clearly stated: e.g., 1 week, 1 month or lifetime? 